\title{
Validação da versão portuguesa de Spiritual Well-Being Questionnaire em idosos institucionalizados
}

\section{Validation of the Portuguese Spiritual Well-Being Questionnaire in institutionalized older adults}

Artigo Original | Original Article

\author{
Mariana Neves, PsyM (1,a), Inês Queiroz Garcia, PsyM (1,b), Helena Espírito-Santo, PhD (1,c), Laura Lemos, PhD (1,d) \\ (1) Instituto Superior Miguel Torga, Coimbra, Portugal \\ (a) Recolha de dados, redação do manuscrito e análise estatística. \\ (b) Redação e revisão do manuscrito e análise estatística. \\ (c) Coordenação do Projeto Trajetórias do Envelhecimento, revisão do manuscrito e análise estatística. \\ (d) Redação e revisão do manuscrito.
}

Autor para correspondência | Corresponding author: Laura Lemos; Largo de Celas, 1, 3000-132 Coimbra, Portugal; +351 910637946; laurastlemos@gmail.com

Palavras-Chave Pessoas idosas Institucionalização Avaliação

SWBQ

Bem-estar espiritual

Keywords

Older adults

Institutionalization

Assessment

SWBQ

Spiritual well-being

\section{RESUMO}

Objetivo: É incontornável a importância que o conceito de bem-estar espiritual assume nos dias de hoje. A investigação é escassa na população idosa institucionalizada, sendo assim importante o desenvolvimento de instrumentos no âmbito do bem-estar espiritual validados para esta população. Este estudo tem como principal objetivo a análise das qualidades psicométricas e a validação da versão portuguesa do Spiritual Well-Being Questionnaire (SWBQ).

Métodos: A amostra incluiu 101 pessoas idosas institucionalizadas da zona de Coimbra $(n=51 ; 50,5 \%)$ e Aveiro $(n=50$; $49,5 \%)$, com idades compreendidas entre os 65 e os 96 anos, 33 (32,7\%) eram do sexo masculino e $68(67,3 \%)$ do sexo feminino. Para além do SWBQ, foram administrados a Escala sobre a Esperança, a Escala de Otimismo, o Geriatric Anxiety Inventory e a Geriatric Depression Scale.

Resultados: O SWBQ apresentou uma estrutura fatorial de três fatores (ambiental, transcendental e humanitário), diferente da versão original cuja estrutura fatorial apontava para quatro fatores. A consistência interna foi adequada e a validade convergente e divergente não atestaram a sua validade de constructo. O bem-estar espiritual foi diferente de forma estatisticamente significativa a nível do sexo $(p<0,01)$, estado civil $(p<0,05)$ e profissão $(p<0,05)$.

Conclusões: Este estudo contribuiu para a disponibilização de um novo instrumento para a avaliação do bem-estar espiritual nas pessoas de idade avançada institucionalizadas, tendo evidenciado características psicométricas razoáveis, devendo, no entanto, ser replicado em amostras com outras características para a confirmação destes dados.

\section{ABSTRACT}

Aims: It is undeniable the importance that the concept of spiritual well-being takes today. Research is scarce in the institutionalized older population, and it is therefore important to develop validated tools on spiritual well-being for this population. The main objective of the present study is the analysis of the psychometric properties and the validation of the Portuguese version of the Spiritual Well-Being Questionnaire (SWBQ).

Methods: The sample included 101 institutionalized older people from the Coimbra area $(n=51,50.5 \%)$ and Aveiro $(n=$ $50,49.5 \%)$, aged 65-96, 33 (32.7\%) were male and 68 (67.3\%) were female. In addition to SWBQ, the Hope Scale, the Optimism Scale, the Geriatric Anxiety Inventory, and the Geriatric Depression Scale were administered.

Results: The SWBQ presented a factorial structure of three factors (environmental, transcendental, and humanitarian), different from the original version whose factor structure pointed to four factors. The internal consistency was adequate and the convergent and divergent validity did not confirm its construct validity. Spiritual well-being was statistically different in terms of gender $(p<0.01)$, marital status $(p<0.05)$ and occupation $(p<0.05)$.

Conclusions: This study contributed to the provision of a new instrument for the evaluation of spiritual well-being in institutionalized older people, showing reasonable psychometric characteristics, but should be replicated in samples with other characteristics to confirm this data. 


\section{INTRODUÇÃO}

O envelhecimento é um processo inevitável que envolve um conjunto de alterações físicas e cognitivas que requerem a adaptação não só do indivíduo, mas também da sociedade (Dhara e Jogsan, 2013; Lima, 2010; Smith e Rush, 2006). Atualmente, no mundo ocidental, assiste-se a um aumento da esperança média de vida e a uma diminuição do número de nascimentos. $O$ índice de envelhecimento em Portugal situava-se, no ano de 2015, em 143,9 (PORDATA, 2015).

O processo de envelhecimento individual é influenciado não só pela idade e biologia, mas também por fatores sociais, económicos, históricos e culturais, revelando-se diferente de indivíduo para indivíduo (Direção-Geral da Saúde, 2017; Schneider e Irigaray, 2008). À medida que a esperança média de vida aumenta, a frequência de doenças ligadas ao envelhecimento tende também a aumentar. Assim, de forma a dar resposta às necessidades que esta realidade impõe, surgem diferentes tipos de resposta social, adequadas às necessidades das pessoas idosas e familiares, tornando-se a institucionalização um fenómeno em crescimento (Chao et al., 2008). Na ausência ou indisponibilidade de familiares, o cuidado de pessoas idosas com diferentes graus de dependência, doença física e/ou défice cognitivo é muitas vezes assegurado por estas instituições havendo cada vez mais uma preocupação com a promoção da saúde e bem-estar.

Neste contexto, para além da importância atribuída à saúde física, psicológica e social surgiu também a ênfase à dimensão espiritual da saúde e o crescente interesse da comunidade científica pelo estudo da espiritualidade e da religiosidade e da sua relação com o bem-estar e saúde mental (Amjad e Bokharey, 2014; Hadzic, 2011; VelascoGonzalez e Rioux, 2014; World Health Organization, 1998). A investigação tem, de facto, indicado que a espiritualidade se relaciona com a qualidade de vida (Ando et al., 2010), bem-estar (Marcoen, 1994) e com a saúde física e mental (Hadzic, 2011; Singh e Kedare, 2014; Velasco-Gonzalez e Rioux, 2014).

Enquanto processo, a espiritualidade é uma vivência contínua e dinâmica (Meraviglia, 1999) sendo vista como uma necessidade de todos os indivíduos (Frankl, 1988) orientando na busca de respostas a questões tão cruciais como o propósito e o significado da vida, a realidade, o amor, o bom, o mau, a doença e a morte (Rahimi, Anoosheh, Ahmadi e Foroughan, 2013).

Ainda assim, a espiritualidade pode ter vários significados e ser entendida de diversas formas por diferentes pessoas (Fisher, 2011), importando distinguir espiritualidade de religiosidade. A religiosidade relacionase com as crenças e práticas simbólicas que aproximam a pessoa do sagrado e a espiritualidade caracteriza-se por uma reflexão pessoal, uma busca pelo significado da vida numa relação com o sagrado ou o transcendente (Crowther, Parker, Achenbaum, Larimore e Koenig, 2002; Kimura, Sakuma, Isaka, Uchida e Yamaoka, 2016; Koenig, 2001; Zinnbauer e Pargament, 2002).

A investigação aponta para a importância da espiritualidade e a sua relação com todos os domínios do bem-estar (Kreitzer, 2012), o que conduziu à criação do conceito de bem-estar espiritual (Gomez e Fisher, 2003; Gouveia, Marques e Pais-Ribeiro, 2009). O bem-estar espiritual engloba questões como o sentido de significado e o propósito; a transcendência; a ligação com o eu, os outros, o mundo e um poder maior; e as buscas espirituais (Lou, 2015). O significado e propósito englobam sentimentos como a paz e a esperança; a transcendência envolve a sensação de bem-estar transtemporal e transespacial; a ligação envolve a sensação de integridade com significado, propósito e esperança; e as buscas espirituais traduzem-se numa sensação ótima do ser e de significado (Lou, 2015).

Em várias definições de bem-estar espiritual está latente a vertente relacional que envolve a ligação consigo mesmo, com os outros e com o transcendente (Gouveia, 2011).

De acordo com Wink e Dillon (2002), não existem estudos longitudinais que abordem o desenvolvimento espiritual na idade adulta, no entanto, o desenvolvimento espiritual parece ocorrer com maior frequência nas fases de transição e crise do que nas fases de maior estabilidade (Stokes, 1990 citado por Wink e Dillon, 2002). Assim, o desenvolvimento espiritual será mais influenciado pelas mudanças sociais e pessoais do que pela idade cronológica (Wink e Dillon, 2002). Apesar disso, estes conceitos estão relacionados, já que ser idoso pode aumentar a probabilidade de experienciar a crise e consequentemente a adversidade (Wink e Dillon, 2002), estando, por exemplo, mais sujeito a vivenciar a morte de um ente querido (Tornstam, 1999) ou a adotar o papel de cuidador perante o cônjuge (Payne, 1994). Ainda, de acordo com Atchley (1997), a ideia de que as etapas mais avançadas do ciclo vital estão relacionadas com o desenvolvimento espiritual pode ser encontrada nas estruturas sociais e culturais das sociedades ocidentais. $\mathrm{Na}$ teoria psicossocial de Erikson (Bester, Naidoo e Botha, 2016) é também defendido que o envelhecimento se caracteriza pela reflexão sobre a vida com o objetivo de perceber se ela foi vivida de uma forma significativa (Bester et al., 2016). Esta procura de significado e sentido da vida conduzirá, por conseguinte, a um maior desenvolvimento espiritual (Nelson, 2009).

Neste sentido, esta investigação tem como principal objetivo o estudo das qualidades psicométricas e 
validação de um instrumento de medição do bem-estar espiritual para pessoas idosas institucionalizadas, designado Spiritual Well-Being Questionnaire.

Este questionário foi desenvolvido por Gomez e Fisher (2003), sendo constituído por 20 itens, distribuídos por quatro subescalas (ambiental, transcendental, comunitária e pessoal), tendo como base o modelo de bem-estar espiritual desenvolvido por Fisher (1999). O Spiritual Well-Being Questionnaire foi validado em quatro estudos realizados com estudantes ingleses, tendo sempre revelado boas qualidades psicométricas (Gomez e Fisher, 2003). O bem-estar espiritual é entendido como um conceito dinâmico que se reflete na qualidade das relações que o indivíduo estabelece nos domínios ambiental, transcendental, comunitário e pessoal. O domínio pessoal refere-se à relação que o indivíduo tem consigo próprio no que concerne ao significado, propósito e valores de vida. O domínio comunitário refere-se às relações interpessoais, sua qualidade e profundidade, em termos de moralidade, cultura e religião. A dimensão ambiental refere-se às relações com o mundo físico e biológico. Por fim, a dimensão transcendental refere-se à relação da pessoa com algo ou alguém para além do humano (Fisher, 1999). De acordo com este autor, o nível de pontuações em cada domínio indica o bem-estar espiritual nessa dimensão (Fisher, 1999). Logo, o bemestar espiritual global resultará do efeito combinado de cada uma das subescalas. Este instrumento é o único que avalia cada uma das dimensões propostas pelo modelo de Fisher, tendo sido este modelo confirmado em outros estudos (Fisher, 2006, 2007; Fisher, Francis e Johnson, 2000, 2002; Gouveia et al., 2009).

Considerando o conceito de espiritualidade de Lou (2015) e de Meraviglia (1999), no estudo da validade de constructo foi considerada para a validade convergente a Escala Sobre a Esperança e a Escala de Otimismo (devido à associação entre o constructo de esperança e o do otimismo). Dadas as relações negativas entre espiritualidade e doença mental (Hadzic, 2011; VelascoGonzalez e Rioux, 2014), para o estudo da validade divergente foi considerado o Geriatric Anxiety Inventory e a Geriatric Depression Scale.

Os estudos realizados para analisar as qualidades psicométricas do Spiritual Well-Being Questionnaire revelaram valores similares. Num dos estudos mais antigos (Gomez e Fisher, 2003), o alfa de Cronbach variou entre 0,74 e 0,90. Numa investigação portuguesa (Gouveia et al., 2009), o alfa de Cronbach situou-se entre 0,72 e 0,89 ( $n=439$; idades: $16-71$ anos). No estudo mais recente (Bester et al., 2016), o alfa de Cronbach do bemestar espiritual global foi de 0,93, variando nos seus quatro fatores entre 0,78 e 0,93 ( $n=122$; idades: $60-69$ anos).

\section{MÉTODO}

\section{Procedimentos}

Este estudo insere-se no Projeto "Trajetórias do Envelhecimento" do Instituto Superior Miguel Torga. Para recolher a amostra foram contactadas oito instituições das zonas de Coimbra e Aveiro, sendo que uma das instituições se recusou a participar no estudo. Todas as instituições foram contactadas primeiro via e-mail e posteriormente presencialmente com o objetivo de apresentar o estudo, assim como de explicar o protocolo que seria administrado. O protocolo foi constituído por um questionário sociodemográfico e 15 instrumentos heteroadministrados: Questionário de Bem-Estar Espiritual (SWBQ), Escala do Arrependimento, Escala sobre a Esperança, Escala de Otimismo, Inventário de Mindfulness de Freiburg, Inventário de Ansiedade Geriátrico (GAI), Escala Geriátrica da Depressão (GDS), Questionário da Saúde Física do Idoso, Escala de Satisfação com a Vida, Lista de Afetos Positivos e Negativos, Questionário sobre o Sono da Terceira Idade, Escala de Auto Compaixão, Escala de Solidão da Universidade da Califórnia, Bateria de Avaliação Frontal e a Avaliação Cognitiva de Montreal.

Após as autorizações por parte das instituições contactadas e das dúvidas esclarecidas, bem como da autorização da autora da versão portuguesa para o uso do instrumento neste estudo, procedeu-se à administração do protocolo. Este protocolo foi aplicado por três mestrandas de psicologia com treino e prática na aplicação de testes neuropsicológicos. Antes de cada administração, foi explicado aos idosos qual o objetivo do estudo e em que consistia o mesmo, assim como foram respondidas todas as dúvidas. O protocolo continha um consentimento informado que era lido a todos os idosos antes da administração dos instrumentos propriamente dita, onde era assegurado o anonimato, a confidencialidade e o caráter voluntário, assim como a possibilidade de desistência a qualquer momento sem qualquer desvantagem para o participante. Não se verificou qualquer caso de recusa por parte dos participantes. A recolha ocorreu entre dezembro de 2015 e setembro de 2016. A administração de cada protocolo demorou aproximadamente de 1 a 2 horas.

\section{Amostra}

Os critérios de inclusão para a amostra do presente estudo foram: ter idade igual ou superior a 65 anos, ter capacidade de entender a linguagem falada (sem afasia sensorial), ter capacidade de falar (sem afasia anterior) e sem demência diagnosticada. Deste estudo foram excluídos 202 idosos por não cumprirem os critérios de inclusão. 
A amostra ficou constituída por 101 pessoas idosas institucionalizadas da zona de Coimbra $(n=51 ; 50,5 \%)$ e Aveiro $(n=50 ; 49,5 \%)$ com idades compreendidas entre os 65 e os 96 anos $(M=81,88 ; D P=7,86)$, sendo que 33 $(32,7 \%)$ eram do sexo masculino e $68(67,3 \%)$ eram do sexo feminino. Sessenta e oito idosos tinham mais de 80 anos (68\%), sendo considerados velhos-velhos. Quanto ao estado civil, verificámos que a maior parte da amostra não tinha companheiro ( $n=80 ; 79,2 \%$ ). Quanto ao nível de escolaridade, a maior parte dos idosos tinha o ensino básico ( $n=48 ; 47,5 \%$ ), seguido dos idosos que não tinham escolaridade ( $n=35 ; 34,7 \%)$. Quanto à profissão, a maioria dos idosos da nossa amostra tinha uma profissão manual ( $n=88$; 88\%) e 12 idosos tinham uma profissão intelectual $(12 \%)$.

\section{Instrumentos}

Para este estudo foram utilizadas cinco medidas de um conjunto de quinze instrumentos.

O Questionário de Bem-estar Espiritual ou Spiritual Well-Being Questionnaire (SWBQ; Gomez e Fisher, 2003; adaptação portuguesa de Gouveia et al., 2009) é um questionário de autopreenchimento constituído por 20 itens distribuídos por quatro subescalas: pessoal, comunitária, ambiental e transcendental. A subescala Pessoal compreende os itens 5 (identidade pessoal), 9 (autoconsciência), 14 (alegria na vida), 16 (paz interior) e 18 (sentido para a vida). A subescala Comunitária compreende os itens 1 (amor pelos outros), 3 (bondade para com os outros), 8 (confiança nos outros), 17 (respeito pelos outros) e 19 (generosidade com os outros). A subescala Ambiental compreende os itens 4 (ligação com a natureza), 7 (admiração da paisagem), 10 (união com a natureza), 12 (harmonia com o ambiente) e 20 (magia na natureza). A subescala Transcendental compreende os itens 2 (relação com Deus), 6 (admiração pela criação), 11 (união com Deus), 13 (paz com Deus) e 15 (vida de meditação ou oração). É pedido ao indivíduo que indique em que medida sente que cada afirmação reflete a sua experiência pessoal atual sendo avaliado numa escala que varia entre "1" (muito pouco), "2" (pouco), "3" (moderadamente), "4" (muito) e "5" (muitíssimo). As pontuações são calculadas através da média das respostas dadas aos itens das subescalas. É também possível calcular a pontuação total do SWBQ através da soma das subescalas. Quanto maior a pontuação, maior o bem-estar espiritual. No que concerne à consistência interna obtida na versão portuguesa, esta foi adequada, com valores de alfa de Cronbach de 0,89 no total do SWBQ e variando entre 0,72 na subescala pessoal e 0,88 na subescala transcendental (Gouveia et al., 2009).

A Escala sobre a Esperança (Barros-Oliveira, 2003) é constituída por seis itens respondidos numa escala tipo Likert de cinco pontos $(1=$ "Totalmente em desacordo" a
$5=$ "Totalmente de acordo"). A pontuação total calculase somando as pontuações das respostas aos itens, variando entre 6 e 30 pontos. São considerados sujeitos com pouca esperança os que obtiverem pontuações inferiores a 15 e com muita esperança os que pontuam acima de 15. Neste estudo a consistência interna foi adequada para efeitos da investigação $(\alpha$ de Cronbach $=$ 0,82), à semelhança do estudo de Barros-Oliveira (2003) que obteve um valor de 0,80 .

A Escala de Otimismo (Barros-Oliveira, 1998) pretende medir o otimismo disposicional através de quatro itens. A resposta é dada numa escala ordinal de 5 posições em formato tipo Likert, variando entre 1 ("totalmente em desacordo") e 5 ("totalmente de acordo"). A pontuação total calcula-se somando as pontuações das respostas aos itens, variando entre 4 e 20 pontos. Neste estudo, a confiabilidade foi elevada $(\alpha$ de Cronbach $=$ 0,88), em conformidade com o estudo original com um alfa de Cronbach de 0,80 (Barros-Oliveira, 2010).

A Escala Geriátrica da Depressão ou Geriatric Depression Scale (GDS; Yesavage et al., 1983; versão portuguesa de Barreto, Leuschner, Santos e Sobral, 2003) é uma escala de heteroavaliação dirigida a idosos. Esta é uma escala composta por 30 itens que medem a presença de sintomas depressivos tendo apenas duas opções de resposta: Sim/Não. Quanto à cotação, atribui-se um ponto a cada resposta positiva nos itens $2,3,4,6,8,10,11,12$, $13,14,16,17,18,20,22,23,24,25,26$ e 28 , bem como a cada resposta negativa nos itens 1, 5, 7, 9, 15, 19, 21, 27, 29 e 30. Assim, a pontuação pode variar entre 0 e 30. Uma pontuação de 0 a 10 é considerada ausência de depressão; entre 11 e 20 pontos é considerada depressão ligeira e entre 21 e 30 pontos é considerada depressão grave. O estudo da validação da escala original obteve boas propriedades psicométricas, tendo o alfa de Cronbach sido de 0,94 (Yesavage et al., 1983). Neste estudo a consistência interna foi considerada adequada para efeitos de investigação ( $\alpha$ de Cronbach $=0,79$ ).

O Inventário de Ansiedade Geriátrica ou Geriatric Anxiety Inventory (GAl; Pachana et al., 2007; versão portuguesa: Espírito Santo e Daniel, 2010) é um instrumento de administração rápida constituído por 20 itens que pretende avaliar a presença de sintomas de ansiedade nas pessoas idosas. É pedido ao indivíduo que indique em que medida concorda ou discorda sobre a sintomatologia ansiógena experienciada na última semana. Os autores da versão original encontraram boas propriedades psicométricas, com um alfa de Cronbach de 0,91 (Pachana et al., 2007). Existem duas validações portuguesas deste instrumento, de Ribeiro, Paúl, Simões e Firmino (2011) e de Espírito Santo e Daniel (2010). No entanto, a confiabilidade neste estudo foi elevada ( $\alpha$ de Cronbach $=0,95)$, à semelhança do estudo de Espírito 
Santo e Daniel (2010), onde foi encontrada uma consistência interna muito boa ( $\alpha$ de Cronbach $=0,93$ ).

\section{Análise estatística}

Com o objetivo de analisar os dados foi utilizado o programa Statistical Package for the Science (SPSS), versão 25 para Macintosh Mavericks. A análise da consistência interna dos instrumentos foi feita através do alfa de Cronbach, considerada a melhor estimativa de fidelidade para um teste (Pallant, 2011). Adicionalmente e para a validade do constructo, foi feita uma análise fatorial do SWBQ.

Procedeu-se a uma análise fatorial exploratória (AFE) com o objetivo de analisar a estrutura dimensional da versão portuguesa do SWBQ. Recorreu-se ao método da análise dos componentes principais (ACP) para a extração dos fatores, seguindo-se uma rotação varimax com normalização de Kaiser. Através da rotação varimax com normalização de Kaiser, os fatores retidos foram os que apresentavam um valor próprio (eigenvalue) superior a 1, estando em consonância com a representação gráfica dos valores próprios e com a análise paralela (Marôco, 2014). Foi utilizado o critério de Kaiser-Meyer-Olkin $(K M O)$ e o teste de esfericidade de Bartlett para avaliar a adequação do modelo AFE. A análise paralela foi feita através do PCA Monte Carlo (Watkins, 2000). Para a validade de constructo, computaram-se as correlações de Pearson e os respetivos coeficientes de determinação $\left(r^{2}\right)$ como estimativas do tamanho do efeito (Espírito-Santo e Daniel, 2017). Finalmente, para a análise da influência das variáveis sociodemográficas sobre o SWBQ, recorremos às correlações ponto bisserial.

\section{RESULTADOS}

\section{Análise fatorial}

Procedeu-se à análise fatorial exploratória com o objetivo de analisar a estrutura dimensional da versão portuguesa do SWBQ. O valor de Kaiser-Meyer-Olkin (KMO) foi de 0,74 , valor que remete para uma boa indicação para a análise fatorial (Marôco, 2014). O teste de esfericidade de Bartlett apresenta um valor de $\chi^{2}=$ 1092,602 $(p<0,001)$, sendo inferior a 0,05, pelo que rejeitamos $\mathrm{H}_{0}$, concluindo que as variáveis estão correlacionadas significativamente. Tendo como base o valor próprio (eigenvalue) superior a 1, a análise paralela de Monte Carlo e o gráfico de sedimentação, foram indicados 3 fatores que explicaram $54 \%$ da variância total. Assim, como mostra a Tabela 1, o Fator 1 (ambiental) ficou constituído pelos itens 4, 7, 10, 12 e 20, explicando $24 \%$ da variância; o Fator 2 (transcendental) pelos itens 2, 6, 11, 13 e 15, explicando 19\% da variância; e, por fim, o Fator 3 pelos itens 1, 3, 17 e 19 da subescala comunitária e pelos itens 14 e 18 da subescala pessoal de acordo com o estudo de validação para a população portuguesa (Gouveia et al., 2009), explicando $11 \%$ da variância. Considerando a natureza dos itens incluídos neste terceiro fator, designámo-lo por fator Humanitário. Como se pode ver na Tabela 1 , face aos baixos pesos fatoriais (< 0,45; Pearson e Hall, 1993), foram excluídos quatro itens desta versão para pessoas idosas (um item da subescala comunitária e três itens da subescala pessoal).

\section{Tabela 1}

Análise de Componentes Principais do Questionário de Bem-estar Espiritual numa Amostra de Pessoas Idosas Institucionalizadas ( $N=101)$

\begin{tabular}{|c|c|c|c|c|}
\hline \multirow{2}{*}{ Item } & \multicolumn{3}{|c|}{ Componentes } & \multirow{2}{*}{$\boldsymbol{h}$} \\
\hline & 1 & 2 & 3 & \\
\hline 1 & & & 0,469 & 0,303 \\
\hline 2 & & 0,860 & & 0,756 \\
\hline 3 & & & 0,648 & 0,470 \\
\hline 4 & 0,876 & & & 0,784 \\
\hline 6 & & 0,774 & & 0,612 \\
\hline 7 & 0,860 & & & 0,746 \\
\hline 10 & 0,919 & & & 0,846 \\
\hline 11 & & 0,865 & & 0,773 \\
\hline 12 & 0,906 & & & 0,833 \\
\hline 13 & & 0,770 & & 0,603 \\
\hline 14 & & & 0,590 & 0,363 \\
\hline 15 & & 0,729 & & 0,593 \\
\hline 17 & & & 0,652 & 0,434 \\
\hline 18 & & & 0,678 & 0,516 \\
\hline 19 & & & 0,769 & 0,600 \\
\hline 20 & 0,897 & & & 0,819 \\
\hline \% de variância & 24 & 19 & 11 & 54 \\
\hline Eigenvalues & 4,71 & 3,78 & 2,24 & \\
\hline
\end{tabular}

Nota. $h^{2}=$ comunalidades. Na tabela são apresentados somente os valores de correlação acima de 0,45 (Pearson e Hall, 1993).

\section{Consistência interna}

Com o objetivo de determinar a consistência interna calculou-se o alfa de Cronbach para todos os itens do questionário e para cada uma das suas dimensões (ambiental, transcendental e humanitário). O SWBQ mostrou ter uma consistência interna adequada (Murphy e Davidshofer, 2004), com valores de alfa de Cronbach de 0,79 no SWBQ Total (16 itens), de 0,95 na subescala ambiental, de 0,87 na subescala transcendental e de 0,68 na subescala humanitário.

\section{Validade convergente}

A análise correlacional para o estudo da validade convergente é apresentada na Tabela 2. 
O SWBQ Total correlacionou-se elevada e significativamente com todas as suas subescalas $\left(r^{2}\right.$ variando entre $26,0 \%$ e $44,9 \%$ ). Nem o SWBQ Total, nem as suas subescalas se correlacionaram de forma estatisticamente significativa com a Escala sobre a Esperança ou com a Escala de Otimismo.

Tabela 2

Correlações de Pearson entre as Pontuações do Spiritual Well-Being Questionnaire (SWBQ), da Escala de Otimismo e da Escala Sobre a Esperança

\begin{tabular}{lllccccc}
\hline & & $\mathbf{1}$ & $\mathbf{2}$ & $\mathbf{3}$ & $\mathbf{4}$ & $\mathbf{5}$ & $\mathbf{6}$ \\
\cline { 2 - 8 } & $\mathbf{1 .}$ Total & - & 0,52 & 0,70 & 0,69 & 0,14 & $-0,05$ \\
\cline { 2 - 8 } SWBQ & $\mathbf{2 .}$ Ambiental & & - & 0,02 & 0,15 & 0,07 & $-0,06$ \\
\cline { 2 - 8 } & 3. Transcendental & & - & 0,20 & 0,10 & $-0,08$ \\
\cline { 2 - 8 } & 4. Humanitário & & & - & 0,13 & 0,08 \\
\hline 5. Otimismo & & & & & - & 0,69 \\
\hline 6. Esperança & & & & & & & - \\
\hline
\end{tabular}

* A correlação é significativa no nível 0,05.

** A correlação é significativa no nível 0,01.

\section{Validade divergente}

A análise correlacional para o estudo da validade divergente é apresentado na Tabela 3.

O SWBQ Total $\left(r^{2}=6,8 \%\right)$ e as subescalas Transcendental $\left(r^{2}=4,0 \%\right)$ e Humanitário $\left(r^{2}=11,6 \%\right)$ correlacionaram-se negativamente de forma estatisticamente significativa, mas fracamente, com a GDS. Nem o SWBQ Total, nem as suas subescalas se correlacionaram de forma estatisticamente significativa com o GAI.

Tabela 3

Correlações de Pearson entre as Pontuações do Spiritual Well-Being Questionnaire (SWBQ), do Geriatric Anxiety Inventory (GAl) e da Geriatric Depression Scale (GDS)

\begin{tabular}{llcccccc}
\hline & $\mathbf{1}$ & $\mathbf{2}$ & $\mathbf{3}$ & $\mathbf{4}$ & $\mathbf{5}$ & $\mathbf{6}$ \\
\cline { 2 - 7 } & 1. Total & - & 0,52 & 0,70 & 0,69 & $-0,02$ & $-0,26^{* *}$ \\
\multirow{2}{*}{ SWBQ } & 2. Ambiental & & - & 0,02 & 0,15 & 0,07 & 0,04 \\
& 3. Transcendental & & - & 0,20 & 0,03 & $-0,20$ \\
& 4. Humanitário & & & - & $-0,15$ & $-0,34$ \\
\hline 5. GAI & & & & - & 0,61 \\
\hline 6. GDS & & & & & - & - \\
\hline
\end{tabular}

* A correlação é significativa no nível 0,05 .

** A correlação é significativa no nível 0,01.

\section{Influência das variáveis sociodemográficas}

O SWBQ Total (sem os itens que tiveram baixa saturação) correlacionou-se significativamente com o sexo $(r=0,30 ; p<0,01)$, o estado civil $(r=-0,25 ; p<0,05)$ e a profissão $(r=-0,21 ; p<0,05)$. A subescala Humanitário correlacionou-se somente com os anos de estudos $\left(r=0,37 ; p<0,01 ; r^{2}=13,7 \%\right)$. A subescala Transcendental correlacionou-se significativamente com o sexo $\left(r=0,43 ; p<0,01 ; r^{2}=18,5 \%\right)$, o estado civil $(r=$ $\left.-0,21 ; p<0,05 ; r^{2}=4,4 \%\right)$ e a profissão $(r=-0,30 ; p<$ 0,$\left.01 ; r^{2}=9,0 \%\right)$. 


\section{DISCUSSÃO E CONCLUSÃO}

O principal objetivo da presente investigação foi o estudo das qualidades psicométricas e a validação de um instrumento de medição do bem-estar espiritual para pessoas idosas institucionalizadas, designado Spiritual Well-Being Questionnaire (SWBQ). Assim, procedeu-se à analise fatorial, à análise da consistência interna, da validade convergente e divergente e da influência das variáveis sociodemográficas.

Com o objetivo de analisar a estrutura dimensional da versão portuguesa do SWBQ recorreu-se à análise fatorial. A estrutura fatorial em estudo apresentou resultados que diferem da validação original e da validação portuguesa. A análise fatorial realizada demonstrou que o modelo a quatro fatores defendido por Gouveia et al. (2009) poderá não ser adequado à população portuguesa idosa e institucionalizada, evidenciando que o modelo a três fatores parece ser o mais adequado para esta população. Estes resultados poderão dever-se às especificidades da população em estudo, já que o estudo de Gouveia et al. (2009) recorreu a população adulta da comunidade.

Quanto à análise da validade convergente, foi escolhido o constructo da esperança uma vez que a revisão bibliográfica apontou para a sua relação com a espiritualidade. A esperança permite que as pessoas confiem no amanhã, acreditem num futuro melhor, sendo, por isso, muitas vezes, considerada como a base da espiritualidade (Davis, 2005), no entanto, não foi encontrada correlação entre esperança e espiritualidade neste estudo.

Ainda no âmbito da análise correlacional para o estudo da validade convergente não se verificou uma relação entre otimismo e bem-estar espiritual, não sendo confirmado o resultado de outros estudos de que pessoas idosas com maiores níveis de espiritualidade são mais otimistas em relação ao futuro (Poor, Borgi, Borgi e Moslemi, 2016).

No que diz respeito à análise correlacional para o estudo da validade divergente, foi possível verificar que o SWBQ Total se correlacionou negativamente, embora de forma fraca, com a GDS. O que significa que pessoas com maiores níveis de bem-estar espiritual apresentam menor sintomatologia depressiva, sublinhando a relação entre a espiritualidade e a saúde mental (Jun, Lee e Bolin, 2015; Koenig e McConnell, 2001).

Relativamente ao GAI, usado também para o estudo da validade divergente, nem o SWBQ Total, nem as suas subescalas se relacionaram com a sintomatologia ansiosa. Este resultado não vai ao encontro da ideia de que pessoas com maiores níveis de espiritualidade parecem mostrar maior tranquilidade apresentando menores índices de ansiedade (Taghiabadi, Kavosi, Mirhafez, Keshvari e Mehrabi, 2017).

Relativamente às variáveis sociodemográficas, foram encontradas diferenças estatisticamente significativas no sexo, estado civil e profissão no bem-estar espiritual.

Quanto ao sexo, verificou-se que as mulheres registaram pontuações mais elevadas do que os homens no bem-estar espiritual. Os dados deste estudo vão ao encontro de outros estudos (Rodrigues et al., 2017; Wink e Dillon, 2002), que defendem que à medida que a idade avança a espiritualidade aumenta, sendo as mulheres idosas a demonstrarem essa preocupação mais cedo e ocorrendo o seu desenvolvimento espiritual a um ritmo mais acelerado do que nos homens. As diferenças nas pontuações do bem-estar espiritual podem dever-se às aprendizagens prévias relacionadas com os papéis de género (Atchley, 1997; Burke, 1999), assim como a diferenças individuais na personalidade da idade adulta jovem (McFadden, 1999). Apesar disso, estes resultados devem ser analisados com cautela já que $67,3 \%$ da amostra é constituída por mulheres.

No que respeita ao estado civil, verificou-se que os indivíduos sem companheiro registaram pontuações mais elevadas de bem-estar espiritual do que os indivíduos com companheiro. Estes dados parecem ir ao encontro de outros estudos (McFadden, 1999; Monteiro, 2009). Este resultado pode dever-se, por um lado, ao facto dos indivíduos com companheiro se apoiarem neste, enquanto que os indivíduos sem companheiro têm de procurar outro tipo de apoio, podendo surgir aqui a espiritualidade como um recurso muito importante (Büssing, Ostermann e Matthiessen, 2005). Por outro lado, as perdas (emocionais, físicas e sociais) na idade adulta avançada parecem favorecer uma maior aproximação à espiritualidade (McFadden, 1999), e, assim, a perda de um companheiro poderá potenciar o desenvolvimento da espiritualidade.

No nosso estudo verificou-se que os indivíduos que tiveram ao longo da vida uma profissão do tipo manual, registaram pontuações mais elevadas de bem-estar espiritual do que os indivíduos com uma profissão intelectual. A investigação aponta para que o estilo de vida na idade adulta jovem, a escolaridade, a ocupação e a posição social, influenciem a forma como envelhecemos (e.g., saúde, bem-estar; Marques, 2003; World Health Organization, 2015), gerando iniquidades no acesso a um estilo de vida saudável e também no acesso a cuidados de saúde. Assim, profissões de tipo manual, socialmente menos valorizadas e com uma retribuição financeira menor, poderão, de alguma forma, contribuir para um envelhecimento com mais problemas de saúde e consequentemente com mais perdas (emocionais, sociais, funcionais). De acordo com os 
dados desta investigação, pessoas com uma profissão de tipo manual e, portanto, potencialmente com mais perdas na idade adulta avançada, apresentam maiores níveis de espiritualidade, indo ao encontro dos estudos que concluem que mais perdas na adultez tardia parecem favorecer uma maior aproximação à espiritualidade, proporcionando maiores níveis de bem-estar (McFadden, 1999; Taghiabadi et al., 2017).

Podem ser apontadas algumas limitações ao presente estudo. A escassez de estudos acerca do bem-estar espiritual na população idosa, especialmente entre as pessoas idosas institucionalizadas, foi uma realidade, pelo que seria importante replicar este estudo com uma amostra significativamente maior. $\mathrm{O}$ facto de incluir apenas pessoas idosas em resposta social será também uma limitação, pelo que seria pertinente, em estudos futuros, incluir também idosos da comunidade. A validade convergente e divergente também não ficou comprovada, havendo necessidade de no futuro este estudo ser replicado com outros instrumentos que atestem a validade de constructo do SWBQ.

Em conclusão, este estudo contribuiu para a disponibilização de um novo instrumento para a avaliação do bem-estar espiritual nas pessoas de idade avançada institucionalizadas, tendo evidenciado características psicométricas razoáveis.

Conflito de interesses | Conflict of interest: nenhum | none. Fontes de financiamento | Funding sources: nenhuma | none.

\section{REFERÊNCIAS}

Amjad, F. e Bokharey, I. Z. (2014). The impact of spiritual wellbeing and coping strategies on patients with generalized anxiety disorder. Journal of Muslim Mental Health, 8(1), 21-38. doi:10.3998/jmmh.10381607.0008.102

Ando, M., Morita, T., Miyashita, M., Sanjo, M., Kira, H. e Shima, Y. (2010). Effects of bereavement life review on spiritual well-being and depression. Journal of Pain and Symptom Management, 40(3), 453-459. doi:10.1016/j.jpainsymman.2009.12.028

Atchley, R. C. (1997). Everyday mysticism: Spiritual development in later adulthood. Journal of Adult Development, 4(2), 123-134. doi:10.1007/bf02510085

Barreto, J., Leuschner, A., Santos, F. e Sobral, M. (2003). Escala de depressão geriátrica. Lisboa: Grupo de Estudos de Envelhecimento Cerebral e Demências.

Barros-Oliveira, J. H. (1998). Optimismo: Teoria e avaliação (proposta de uma nova escala) Psicologia, Educação e Cultura, 2(2), 295308

Barros-Oliveira, J. H. (2003). Esperança: Natureza e avaliação (proposta de uma nova escala). Psicologia, Educação e Cultura, 7(1), 83-106. Disponível em http://hdl.handle.net/10216/94145

Barros-Oliveira, J. H. (2010). Felicidade, otimismo, esperança e perdão em jovens, adultos e idosos. Psychologica, 52(1), 123-147. doi:10.14195/1647-8606_52-1_7

Bester, E., Naidoo, P. e Botha, A. (2016). The role of mindfulness in the relationship between life satisfaction and spiritual wellbeing amongst the elderly. Social Work, 52(2), 245-266. doi:10.15270/52-2-503
Burke, P. C. (1999). Spirituality: A continually evolving component in women's identity development. Em L. E. Thomas e S. A. Eisenhandler (Eds.), Religion, belief, and spirituality in late life (pp. 113-136). New York, NY: Springer.

Büssing, A., Ostermann, T. e Matthiessen, P. F. (2005). The role of religion and spirituality in medical patients in Germany. Journal of Religion and Health, 44(3), 321-340. doi:10.1007/s10943-0055468-8

Chao, S.-Y., Lan, Y.-H., Tso, H.-C., Chung, C.-M., Neim, Y.-M. e Clark, M. J. (2008). Predictors of psychosocial adaptation among elderly residents in long-term care settings. The Journal of Nursing Research, 16(2), 149-159. doi:10.1097/01.JNR.0000387300.22172.c6

Crowther, M. R., Parker, M. W., Achenbaum, W. A., Larimore, W. L. e Koenig, H. G (2002). Rowe and Kahn's model of successful aging revisited: Positive spirituality - the forgotten fator. The Gerontologist, 42(5), 613-620. doi:10.1093/geront/42.5.613

Davis, B. (2005). Mediators of the relationship between hope and wellbeing in older adults. Clinical Nursing Research, 14(3), 253-272. doi:10.1177/1054773805275520

Dhara, R. D. e Jogsan, Y. A. (2013). Depression and psychological wellbeing in old age. Journal of Psychology \& Psychotherapy, 3(3), 117-120. doi:10.4172/2161-0487.1000117

Direção-Geral da Saúde. (2017). Estratégia nacional para o envelhecimento ativo e saudável: 2017-2025. Lisboa: Ministério da Saúde. Recuperado de https://www.sns.gov.pt/wpcontent/uploads/2017/07/ENEAS.pdf

Espírito-Santo, H. e Daniel, F. (2017). Calcular e apresentar tamanhos do efeito em trabalhos científicos (2): Guia para reportar a força das relações. Revista Portuguesa de Investigação Comportamental e Social, 3(1), 53-64. doi:10.7342/ismt.rpics.2017.3.1.48

Espírito Santo, H. e Daniel, F. (2010). Inventário de Ansiedade Geriátrica [Instrumento de avaliação] [Geriatric Anxiety Inventory]. Instrumento não publicado. Coimbra: Instituto Superior Miguel Torga.

Fisher, J. W. (1999). Helps to fostering students' spiritual health. International Journal of Children's Spirituality, 4(1), 29-49. doi:10.1080/1364436990040104

Fisher, J. W. (2011). The four domains model: Connecting spirituality, health and well-being. Religions, 2(1), 17-28. doi:10.3390/rel2010017

Fisher, J. W., Francis. L. J. e Johnson, P. (2000). Assessing spiritual health via four domains of spiritual wellbeing: The SH4DI. Pastoral Psychology, 49(2), 133-145.

Fisher, J. W., Francis, L. J. e Johnson, P. (2002). The personal and socia correlates of spiritual well-being among primary school teachers. Pastoral Psychology, 51(1), 3-11.

Fisher, J. W. (2006). Using secondary students' views about influences on their spiritual well-being to inform pastoral care. International Journal of Children's Spirituality, 11(3), 347-356.

Fisher, J. W. (2007). It's time to wake up and stem the decline in spiritual well-being in Victorian schools. International Journal of Children's Spirituality, 12( 2), 165-177.

Frankl, V. E. (1988). The will to meaning: Foundations and applications of logotherapy. New York, NY: Penguin, Expanded edition.

Gomez, R. e Fisher, J. W. (2003). Domains of spiritual well-being and development and validation of the spiritual well-being questionnaire. Personality and Individual Differences, 35(8), 19751991. doi:10.1016/s0191-8869(03)00045-x

Gouveia, M. J. P. M. (2011). Flow disposicional e o bem-estar espiritual em praticantes de actividades físicas de inspiração oriental. [Tese de doutoramento]. Instituto Universitário de Ciências Psicológicas, Sociais e da Vida, Universidade do Porto. Disponível em http://hdl.handle.net/10400.12/1226

Gouveia, M. J., Marques, M. e Pais-Ribeiro, J. L. (2009). Versão portuguesa do questionário de bem-estar espiritual (SWBQ): Análise confirmatória da sua estrutura factorial. Psicologia, Saúde \& Doenças, 10(2), 285-293.

Hadzic, M. (2011). Spirituality and mental health: Current research and future directions. Journal of Spirituality in Mental Health, 13(4), 223-235. doi:10.1080/19349637.2011.616080

Jun, J. S., Lee, K. H. e Bolin, B. L. (2015). Stress and spirituality on the 
depressive symptoms of older adults in assisted living: Gender differences. Journal of Evidence-Informed Social Work, 12(6), 588600. doi:10.1080/15433714.2014.966229

Kimura, T., Sakuma, T., Isaka, H. Uchida, S. e Yamaoka, K. (2016). Depressive symptoms and spiritual wellbeing in Japanese university students. International Journal of Culture and Mental Health, 9(1), 14-30. doi:10.1080/17542863.2015.1074261

Koenig, H. G. (2001). Religion, spirituality, and medicine: How are they related and what does it mean? Mayo Clinic Proceedings, 76(12), 1189-1191. doi:10.4065/76.12.1189

Koenig, H. G. e McConnell, M. (2001). The healing power of faith: How belief and prayer can help you triumph over disease. London, UK: Simon \& Schuster.

Kreitzer, M. J. (2012). Spirituality and well-being: Focusing on what matters. Western Journal of Nursing Research, 34(6), 707-711. doi:10.1177/0193945912448315

Lima, M. P. (2010). Envelhecimento(s) (1. ${ }^{\mathrm{a}}$ ed.). Coimbra: Imprensa da Universidade de Coimbra.

Lou, V. W. Q. (2015). Spiritual well-being of Chinese older adults: Conceptualization, measurement and intervention (1. ${ }^{\mathrm{a}}$ ed.). Heidelberg: Springer. doi:10.1007/978-3-662-46303-1

Marcoen, A. (1994). Spirituality and personal well-being in old age. Ageing and Society, 14(4), 521-536. doi:10.1017/S0144686X00001896

Marôco, J. (2014). Análise estatística com o SPSS statistics (6. ${ }^{a}$ ed.). Pêro Pinheiro: ReportNumber.

Marques, L. F. (2003). A saúde e o bem-estar espiritual em adultos portoalegrenses. Psicologia, Ciência e Profissão, 23(2), 56-65. doi:10.1590/S1414-98932003000200009

McFadden, S. H. (1999). Religion, personality, and aging: A life span perspective. Journal of Personality, 67(6), 1081-1104. doi:10.1111/1467-6494.00083

Meraviglia, M. G. (1999). Critical analysis of spirituality and its empirical indicators: Prayer and meaning in life. Journal of Holistic Nursing, 17(1), 18-33. doi:10.1177/089801019901700103

Monteiro, M. R. (2009). Correlatos de bem-estar espiritual de indivíduos em reabilitação cardíaca pós-enfarte agudo do miocárdio (Dissertação de mestrado]. Universidade Fernando Pessoa, Porto. Obtido em http://hdl.handle.net/10284/1216

Murphy, K. R. e Davidshofer, C. O. (2004). Psychological testing: Principles and applications (6. ${ }^{\mathrm{a}}$ ed.). Englewood Cliffs, NJ: Prentice-Hall.

Nelson, J. M. (2009). Psychology, religion, and spirituality (1. ${ }^{\mathrm{a}}$ ed.). New York, NY: Springer-Verlag.

Pachana, N. A., Byrne, G. J., Siddle, H., Koloski, N., Harley, E. e Arnold, E. (2007). Development and validation of the Geriatric Anxiety Inventory. International Psychogeriatrics, 19(1), 103-114. doi:10.1017/S1041610206003504

Pallant, J. (2011). SPSS survival manual: A step by step guide to data analysis using SPSS for Windows $\left(4 .^{\mathrm{a}}\right.$ ed.). Crows Nest NSW: Allen and Unwin.

Payne, B. P. (1994). Faith development of older men. Em E. H. Thompson (Ed.), Older men's lives (pp. 85-103). Thousand Oaks, CA: Sage.

Pearson, L. C. e Hall, B. W. (1993). Initial construct validation of the Teaching Autonomy Scale. Journal of Educational Research, 86(3), 172-178. doi:10.1080/00220671.1993.9941155

Poor, H. J., Borgi, M., Borgi, M. e Moslemi, A. (2016). The relationship between spiritual well-being and quality of life and optimism on the staff of Arak University of Medical Sciences. Health, Spirituality and Medical Ethics, 3(2), 8-15.
PORDATA. (2015). Índice de envelhecimento: Onde há mais e menos idosos por 100 jovens? Base de dados Portugal Contemporâneo. Recuperado https://www.pordata.pt/Municipios/\%C3\%8Dndice+de+envelhec imento-458

Rahimi, A., Anoosheh, M., Ahmadi, F. e Foroughan, M. (2013). Exploring spirituality in Iranian healthy elderly people: A qualitative conten analysis. Iranian Journal of Nursing and Midwifery Research, 18(2), 163-170.

Ribeiro, O., Paúl, C., Simões, M. R. e Firmino, H. (2011). Portuguese version of the Geriatric Anxiety Inventory: Transcultural adaptation and psychometric validation. Aging \& Mental Health 15(6), 742-748. doi:10.1080/13607863.2011.562177

Rodrigues, L. R., Nader, I. D., Silva, A. T. M., Tavares, D. M. S. Assunção, L. M. e Molina, N. P. F. M. (2017). Spirituality and religiosity related to socio-demographic data of the elderly population. Northeast Network Nursing Journal, 18(4), 429-436. doi:10.15253/2175-6783.2017000400002

Schneider, R. H. e Irigaray, T. Q. (2008). O envelhecimento na atualidade: Aspectos cronológicos, biológicos, psicológicos e sociais. Estudos de Psicologia (Campinas), 25(4), 585-593. doi:10.1590/S0103-166X2008000400013

Singh, D. e Kedare, J. (2014). A study of depression in medically il elderly patients with respect to coping strategies and spirituality as a way of coping. Journal of Geriatric Mental Health, 1(2), 83-89. doi:10.4103/2348-9995.152427

Smith, G. e Rush, B. K. (2006). Normal aging and mild cognitive impairment. Em D. K. Attix e K. A. Welsh-Bohmer (Eds.), Geriatric neuropsychology: Assessment and intervention (pp. 27-55). New York, NY: Guilford Press.

Taghiabadi, M., Kavosi, A., Mirhafez, S. R., Keshvari, M. e Mehrabi, T. (2017). The association between death anxiety with spiritual experiences and life satisfaction in elderly people. Electronic Physician, (9)3, 3980-3985. doi:10.19082/3980

Tornstam, L. (1999). Late-life transcendence: A new developmental perspective on aging. Em L. E. Thomas e S.A. Eishenhandler (Eds.) Religion, belief, and spirituality in late life (pp. 178-202). New York, NY: Springer.

Velasco-Gonzalez, L. e Rioux, L. (2014). The spiritual well-being of elderly people: A study of a French sample. Journal of Religion and Health, 53(4), 1123-1137. doi:10.1007/s10943-013-9710-5

Watkins, M. W. (2000). Monte Carlo PCA for parallel analysis [Programa Informático]. State College, PA: Ed and Psych Associates.

Wink, P. e Dillon, M. (2002). Spiritual development across the adult course: Findings from a longitudinal study. Journal of Adul Development, 9(1), 79-94. doi:1068-0667/02/0100-0079/0

World Health Organization. (1998). WHOQOL and spirituality, religiousness and personal beliefs (SRPB). [WHO reference number: WHO/MSA/MPH/98.2]. Disponível em http://www.who.int/iris/handle/10665/70897

World Health Organization. (2015). World report on ageing and health. Genebra: Autor.

Yesavage, J. A , Brink, T. L., Rose, T. L., Lum, O, Huang, V., Adey, M e Leirer, V. O. (1983). Development and validation of a geriatric depression screening scale: A preliminary report. Journal of Psychiatric Research, 17(1), 37-49. doi:10.1016/00223956(82)90033-4

Zinnbauer, B. J. e Pargament, I. K. (2002). Capturing the meanings of religiousness and spirituality: One way down from a definitional Tower of Babel. Em R. L. Piedmont e D. O. Moberg, Research in the social scientific study of religion (Vol. 13, pp. 23-54). 